\title{
Rab1A promotes proliferation and migration abilities via regulation of the HER2/AKT-independent mTOR/S6K1 pathway in colorectal cancer
}

\author{
ZHENGWU CHENG $^{1 *}$, XINYU SHAO ${ }^{2,3 *}$, MENGLIN XU ${ }^{4}$, JUNFENG WANG $^{1}$, XIAOYI KUAI ${ }^{2}$, \\ LIPING ZHANG ${ }^{2}$, JIAN WU ${ }^{1}$, CHUNLI ZHOU ${ }^{2}$ and JIADING MAO ${ }^{1}$
}

\begin{abstract}
${ }^{1}$ Department of Gastrointestinal Surgery, The First Affiliated Hospital of Wannan Medical College, Wuhu, Anhui 241000; Departments of ${ }^{2}$ Gastroenterology and ${ }^{3}$ General Surgery, The Affiliated Suzhou Hospital of Nanjing Medical University, Suzhou, Jiangsu 215006; ${ }^{4}$ Department of Oncology, The First Affiliated Hospital of Wannan Medical College, Wuhu, Anhui 241000, P.R. China
\end{abstract}

Received February 14, 2019; Accepted March 7, 2019

DOI: 10.3892/or.2019.7071

\begin{abstract}
Colorectal carcinoma (CRC) is one of the most common malignancies worldwide and the second leading cause of cancer-related deaths in the US. Recently, Rab1A has been reported to be an activator of $\mathrm{mTORC} 1$ and $\mathrm{p}-\mathrm{S} 6 \mathrm{~K} 1$, which is downstream of mTORC1. However, the association between Rab1 A and p-S6K1 in CRC remains elusive. In the present study, we first demonstrated that RablA was overexpressed in CRC tissues and Rab1A overexpression was positively related to lymph node invasion, degree of differentiation, venous invasion and tumor-node-metastasis (TNM) stage. In both TNM stage I-II and III-IV patients, Rab1A-positive patients had a shorter survival time than Rab1A-negative patients. Furthermore, in univariate and multivariate analyses, only Rab1A expression was verified as an independent prognostic factor for survival in CRC patients. The level of p-S6K1 was markedly high in CRC tissues and Rab1A expression level had a positive association with p-S6K1 level. In addition, high levels of both Rab1A and p-S6K1 were associated with a poorer prognosis compared with low expression of either Rab1A or p-S6K1 level. Moreover, high levels of both Rab1A and p-S6K1 were associated with a poorer prognosis than patients with high levels of either Rab1A or p-S6K1 alone.
\end{abstract}

Correspondence to: Dr Jiading Mao, Department of Gastrointestinal Surgery, The First Affiliated Hospital of Wannan Medical College, 2 Zheshan West Road, Jinghu, Wuhu, Anhui 241000, P.R. China

E-mail: maojiadingyjs02@126.com

Dr Chunli Zhou, Department of Gastroenterology, The Affiliated Suzhou Hospital of Nanjing Medical University, 242 Guangji Road, Suzhou, Jiangsu 215006, P.R. China

E-mail: zhouchunli079@163.com

${ }^{*}$ Contributed equally

Key words: colorectal cancer, Rab1A, p-S6K1, mTORC1, prognosis
Finally, knockdown of Rab1A expression inhibited migration and proliferation of SW480 and HCT116 cell lines by targeting regulation of $\mathrm{p}-\mathrm{S} 6 \mathrm{~K} 1$. Thus, our findings indicate that Rab1 A plays an important role in $\mathrm{CRC}$ and may provide a therapeutic target for CRC, particularly for mTORC1-targeted therapy-resistant cancers.

\section{Introduction}

Cancer is a major public health problem worldwide and is the second leading cause of cancer-related deaths in the US (1). There has been a rise in the incidence and mortality rates of colorectal cancers (CRC) in Asia (2). To date, complex therapies including surgery, chemotherapy and molecular targeted therapy have been used to treat CRC widely. However, the prognosis remains relatively poor, mainly due to metastasis to distant organs (3). Therefore, newer therapeutic targets are urgently required to improve the survival of the patients.

RablA is a member of the RAB family, a small GTPase, that mediates vesicular trafficking from the endoplasmic reticulum (ER) to the Golgi apparatus (4). Rab1A protein is also involved in mediating signal transduction (5), cell migration (6) and regulation of autophagy (7). Aberrant expression of Rab1A has been linked to a range of human diseases, including Parkinson's disease and cardiomyopathy $(8,9)$. Recently, varying levels of Rab1A overexpression have been reported in many malignant tumors such as breast cancer (10), and hepatocellular carcinomas (HCC) (11). In addition, Rab1A overexpression was revealed to play a significant role in the development of different tumors. Rab1A has been revealed to function as an activator of mTORC1 in HCC (11). Thomas et al reported overexpression of Rab1 A in CRC, which was revealed to be correlated with elevated mTORC1 signaling, tumor invasion, progression, and poor prognosis (12).

Mammalian target of rapamycin (mTOR), as a downstream effector in the PI3K/AKT signaling pathway, consists of two distinct complexes designated mTORC1 and mTORC2, which are composed of the mTOR kinase subunit and accessory proteins (13). As part of a complicated pathway, mTORC1 
plays various roles in cell survival, growth, metabolism, and cell cycle progression and is sensitive to rapamycin (14). Due to the central role of mTORC1 in growth, aberrant mTORC1 signaling has been linked to human diseases, particularly cancer (15). mTORC1 activation promotes cell survival through the activation of anti-apoptotic proteins, thus contributing to tumor progression $(16,17)$. Despite the increasing number of mTORC1-targeted clinical trials, development to date has been limited (18).

Rab1A functions as an oncogene and an activator of mTORC1 in HCC and CRC $(11,12)$. The link between Rab and $\mathrm{p}$-S6K1, which is the main signaling molecule downstream of mTORC1, was first reported by Li et al (19) in 2010; however, the relationship between p-S6K1 and Rab1 A in CRC remains to be fully elucidated. Therefore, in the present study, we examined the association between Rab1A and p-S6K1 levels in CRC specimens to determine their prognostic significance. Moreover, we validated the anticancer effects of Rab1A knockdown in CRC cells to evaluate the importance of Rab1A as a novel therapeutic target in CRC, particularly for mTORC1-targeted therapy-resistant cancers.

\section{Patients and methods}

Human colorectal cancer tissues and colorectal cancer cell lines. CRC tissues were obtained from 142 patients undergoing radical surgery for CRC at the Department of General Surgery, the Affiliated Suzhou Hospital of Nanjing Medical University (Suzhou, China) from 2008-2013. The diagnosis of colorectal adenocarcinoma was confirmed by pathological examination. None of the patients received radiotherapy or chemotherapy prior to surgery. All patients had complete clinical data and were available for follow-up. This study was approved by the Independent Ethics Committee of the Affiliated Suzhou Hospital of Nanjing Medical University and all participants provided written informed consent. After surgery, each patient was followed-up regularly. Human CRC cell lines SW480 (from a primary adenocarcinoma of the colon; adherent cells; tumorigenic: yes), LOVO (derived from metastatic site: left supraclavicular region; adherent cells; tumorigenic: yes), and RKO (a poorly differentiated colon carcinoma cell line; adherent cells; tumorigenic: yes) were purchased from the Chinese Academy of Sciences (Shanghai, China), and the HCT116 (derived from the parental HCT116 colorectal carcinoma cell line; adherent cells) and COLO201 (derived from metastatic site: ascites; suspension, with some loosely adherent cells; tumorigenic: yes) were obtained from the College of Life Sciences, Soochow University (Suzhou, China) mainly selected due to the fact that they are relatively representative and have different characteristics. All five cell lines were maintained in Dulbecco's modified Eagle's medium supplemented with $10 \%$ fetal bovine serum (Gibco; Thermo Fisher Scientific, Inc., Waltham, MA, USA) and cultured at $37^{\circ} \mathrm{C}$ in a humidified atmosphere containing $5 \% \mathrm{CO}_{2}$.

Immunohistochemistry (IHC). Rab1A protein expression was analyzed in 142 CRC tissue samples and 40 adjacent normal colorectal tissue samples by immunohistochemical staining (IHC). The surgical specimens were fixed in $10 \%$ formalin and embedded in paraffin and serial sections (thickness, $5 \mu \mathrm{m}$ ) were prepared. Sections were then dewaxed, rehydrated and endogenous peroxidase activity was blocked by incubation with peroxide in methanol. Non-specific immunoglobulin binding was blocked by incubation with $10 \%$ normal goat serum for $15 \mathrm{~min}$. After washing with phosphate-buffered saline (PBS), sections were incubated with the polyclonal antibodies for the detection of Rab1A (dilution 1:100; cat. no. 11671-1-AP; ProteinTech; Wuhan Sanying Biotechnology, Wuhan, China), p-AKT (dilution 1:100; cat. no. 4060; Cell Signaling Technology, Inc., Danvers, MA, USA), HER2 (dilution 1:100; cat. no. ab131490) and p-S6K1 (dilution 1:100; cat. no. ab60948) (both from Abcam, Cambridge, UK) at room temperature for 2-3 $\mathrm{h}$ and stained using a tissue staining kit (Beijing Zhongshan Jinqiao Biotechnology Co., Ltd., Beijing, China) according to the manufacturer's protocol. Immunostaining was examined independently by two clinical pathologists who were blinded to the patient outcome. Five high-power fields (x200, magnification) in each section were randomly selected for analysis according to the percentage of cells stained and the intensity of the staining. The percentage of positively staining cells per section was scored as follows: Absent, 0-5\%; 1, 6-25\%; 2, 26-50\%; 3, 51-75\%; 4, >75\%. The staining intensity was scored as follows: 0 (negative); 1 (weak); 2 (moderate); 3 (strong). The percentage and intensity scores were then multiplied to achieve a total score (staining score $=$ percentage score $\mathrm{x}$ intensity score). The total staining score was classified as follows: $0,(-) ; 1-4,(+)$; $5-8,(++), 9-12,(+++)$. In the present study, we classified all of the samples into the high expression group $(++$ or +++$)$ and the low expression group (- or + ) according to the protein expression. The IHC method and staining score were performed as described by Yang et al and Shao et al $(20,21)$.

\section{Short hairpin RNA transfection of human colorectal cancer cell lines.SW480/HCT116 celllines stably expressing Rab1 A-specifc shRNA or scrambled control shRNA were generated using a lentiviral shRNA transfection technique. The sequences specific for human Rab1 A (5'-CAGCAUGAAUCCCGAAUAUTT-3') selected to inhibit the Rab1A gene expression were synthesized by (Shanghai GenePharma Co., Ltd., Shanghai, China). SW480 and HCT116 cells were transfected with shRab1A or control shRNA using Lipofectamine 2000 (Invitrogen; Thermo Fisher Scientific, Inc.) according to the manufacturer's instructions. At $72 \mathrm{~h}$ post-transfection, cells were subjected to puromycin selec- tion to obtain a stable cell line. The efficiency of Rab1A-shRNA was detected by fluorescence microscopy and western blot analysis.}

Protein extraction and western blot analysis. Whole protein extracts from SW480/HCT116 were lysed for $30 \mathrm{~min}$ in ice-cold RIPA lysis buffer (Beyotime Biotechnology, Inc., Nantong, China) according to the manufacturer's protocol. Proteins were separated by SDS-PAGE with the quantity of $10 \mu \mathrm{g}$, and then transferred to the nitrocellulose membranes (Schleicher \& Schuell BioScience, Inc., Keene, NH, USA). Standard western blotting was performed using a polyclonal rabbit antibody against human Rab1A (dilution 1:2,000; cat. no. ab97956), rabbit anti-human p-S6K1 (dilution 1:1,000; cat. no. ab60948) (both from Abcam), rabbit anti-human p-AKT (dilution 1:1,000; cat. no. 4060; Cell Signaling 
A
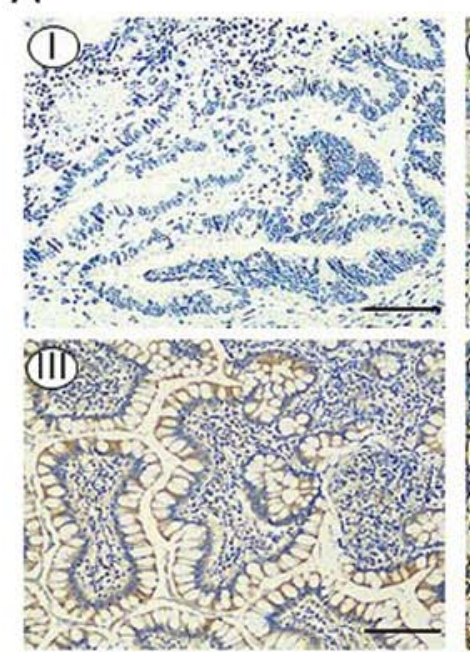
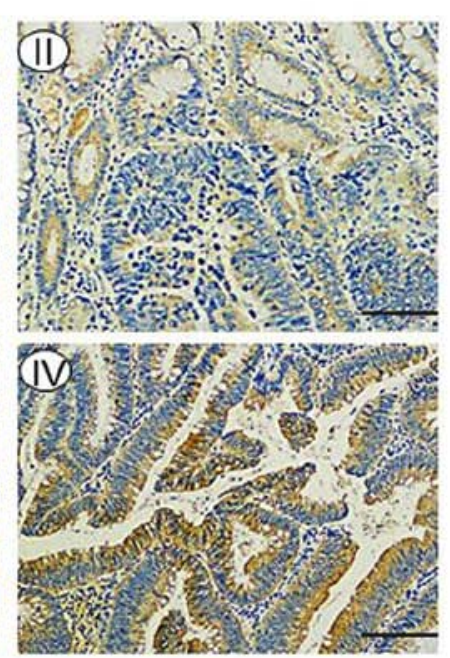

B

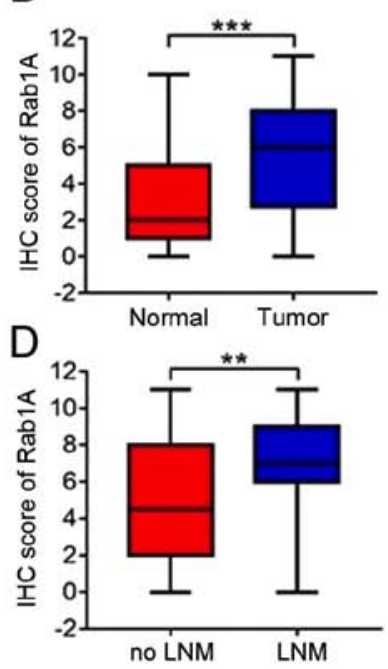

C

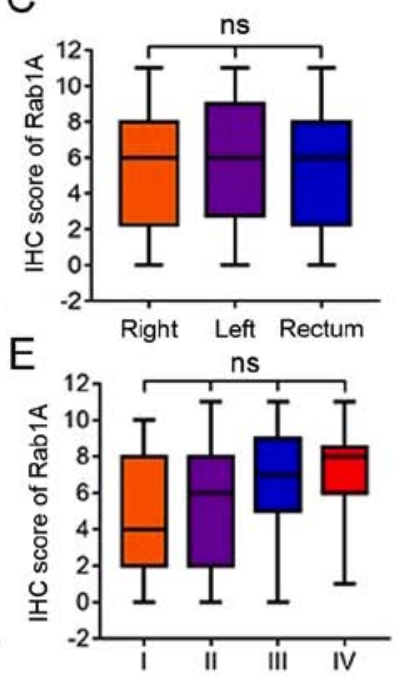

Figure 1. Expression of Rab1A in CRC tissues. (A) Immunohistochemical (IHC) staining of Rab1A in 142 pairs of primary human CRC and adjacent normal tissues (magnification, x200) (scale bar, $100 \mu \mathrm{m}$ ). The expression of Rab1A protein was negative (I), weak (II), positive (III), or strong positive (IV). (B) Staining scores of Rab1A expression in CRC tissues and adjacent normal tissues. (C) Staining scores of Rab1A expression in right colon carcinoma, left colon carcinoma and rectal carcinoma. (D) Staining scores of Rab1A expression in lymph node invasion-positive and negative patients. (E) Staining scores of Rab1A expression in four TNM stages. ${ }^{* *} \mathrm{P}<0.01,{ }^{* * * *} \mathrm{P}<0.001$. CRC, colorectal carcinoma; TNM, tumor-node-metastasis; LNM, lymph node metastasis.

Technology, Inc., Danvers, MA, USA), rabbit anti-human HER2 (dilution 1:1,000; cat. no. ab131490; Abcam), rabbit anti-human AKT (dilution 1:1,000; cat. no. 9272; Cell Signaling Technology), rabbit anti-human S6K1 (dilution 1:1,000; cat. no. ab32359; Abcam) and mouse anti-human GAPDH antibody (dilution 1:5,000; cat. no. AF7021; Affinity Biosciences, Cincinnati, OH, USA). After blocking with $5 \%$ non-fat milk for $1 \mathrm{~h}$ at room temperature, the membranes were incubated overnight with primary detection antibodies and then with conjugated secondary detection antibodies for $1 \mathrm{~h}$ at room temperature. Immunoreactive bands were visualized by chemiluminescence and quantified using Image J software (version 1.4.3.67; NIH; National Institutes of Health, Bethesda, MD, USA).

MTT assays of cell viability. Cell viability was analyzed using MTT assay kits (Amresco, LLC, Solon, OH, USA) according to the manufacturer's protocol. Cells were digested, resuspended, and inoculated into 96-well culture plates. After incubation in complete medium, MTT solution was added and cells were incubated at $37^{\circ} \mathrm{C}$ for $4 \mathrm{~h}$. The supernatants were then removed, and formazan crystals were dissolved in $150 \mu 1$ dimethyl sulfoxide (DMSO). After incubation for $10 \mathrm{~min}$ at $37^{\circ} \mathrm{C}$, the absorbance at $490 \mathrm{~nm}$ was measured. For each sample, five replicate wells were prepared, and the experiment was repeated three times.

Cell migration assay. The migratory activity of the Rab1A or control shRNA-transfected SW480 cells/HCT116 cells was evaluated in 24-well Transwell plate assays (Corning Incorporated, Corning, NY, USA) according to the manufacturer's protocol. Cells resuspended in serum-free RPMI-1640 medium were plated in the upper chamber of each well. Each lower chamber was filled with $500 \mu \mathrm{l}$ complete culture medium. After $24 \mathrm{~h}$ of incubation at $37^{\circ} \mathrm{C}$, the cells on the upper side of the membrane insert were completely removed by wiping with a cotton swab. The cells that had invaded were fixed in methanol, stained with $0.5 \%$ crystal violet and counted manually in five randomly selected fields under an inverted microscope (scale bar, $200 \mu \mathrm{m}$ ). Each experiment was repeated in triplicate.

Statistical analysis. All experiments were repeated three times. Spearman's correlation was used to measure the correlation between Rab1 A and p-S6K1 IHC scores. Data were expressed as the means \pm standard error of the mean (SEM). Significant differences between the groups were determined using Student's t-test and the Chi-square test. Survival duration was calculated using the Kaplan-Meier method and compared using the log-rank test. All parameters that were discovered to be significant on univariate analysis with the Cox proportional hazard model were then included in the multivariate survival analysis. A value of $\mathrm{P}<0.05$ was considered to indicate a statistically significant difference. All statistical analyses were performed with SPSS 17.0 software (SPSS, Inc., Chicago, IL, USA) and GraphPad Prism 5 (GraphPad Software, Inc., La Jolla, CA, USA). The images were combined using Microsoft PowerPoint (PPT).

\section{Results}

RablA expression levels in colorectal cancer tissues and paired adjacent tissues. We investigated the expression of Rab1A in 142 pairs of primary human CRC and adjacent normal tissues by IHC staining. The percentage staining and intensity scores were then multiplied to achieve a total staining score (Fig. 1A). The results indicated that the Rab1A expression levels were significantly higher in CRC than in paracancer tissues $(\mathrm{P}<0.001$; Fig. 1B). Furthermore, Rab1A expression was significantly higher in patients with lymph node invasion compared with those without $(\mathrm{P}<0.01$; Fig. 1D). However, there were no significant differences in Rab1A expression between right 
A

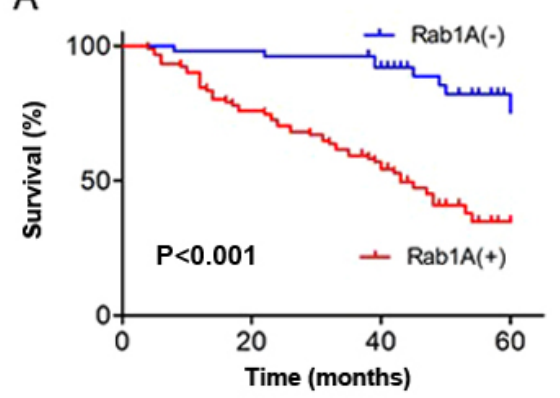

D

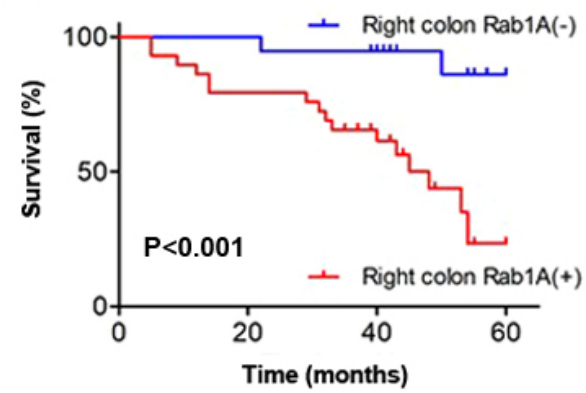

B

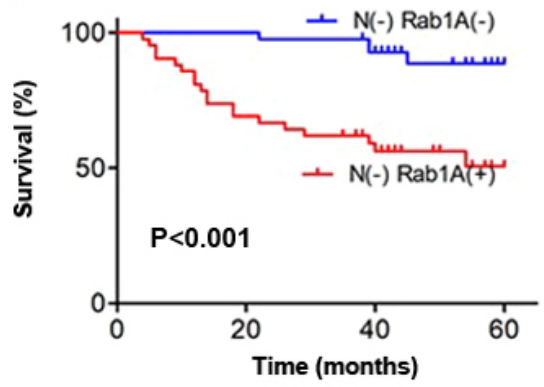

E

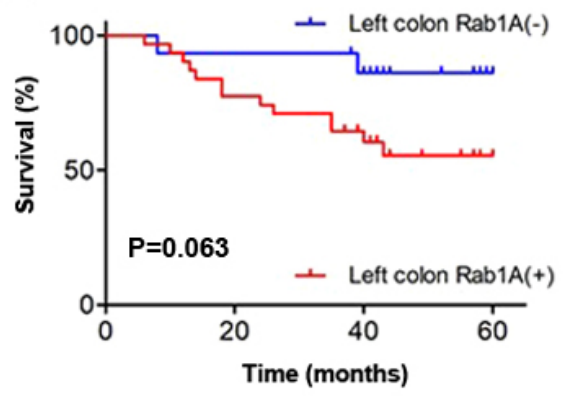

C

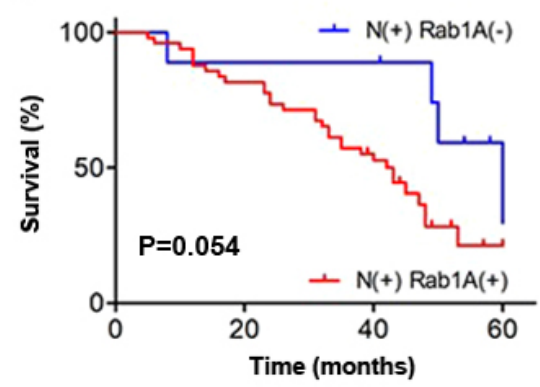

$\mathrm{F}$

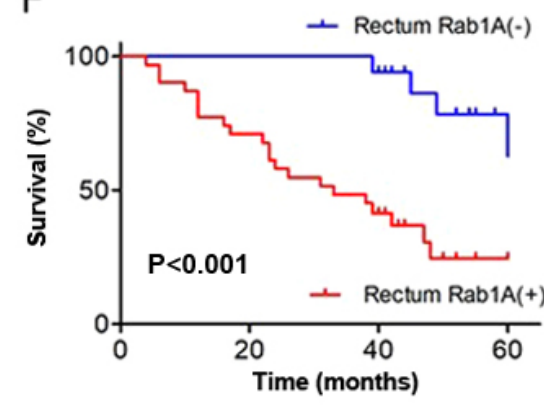

G

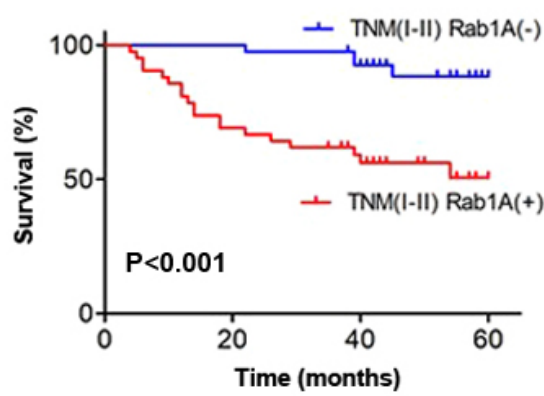

$\mathrm{H}$

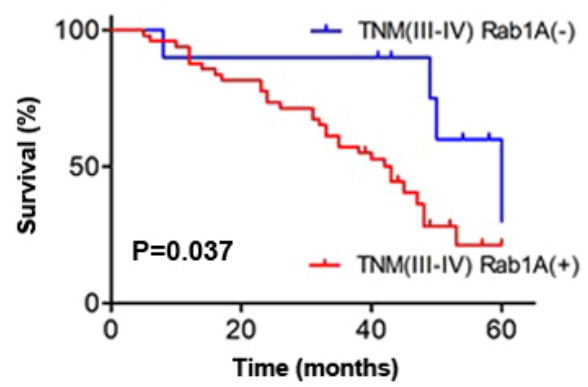

Figure 2. Kaplan-Meier survival analysis of patients after radical resection of colorectal cancer (CRC). (A) The overall survival (OS) of 142 CRC patients after surgery was analyzed according to Rab1A expression. Survival analysis according to (B) negative lymph node invasion and (C) positive lymph node invasion and Rab1A expression. Survival analysis according to different tumor locations: (D) right colon carcinoma, (E) left colon carcinoma and (F) rectal carcinoma and Rab1A expression. Survival analysis of TNM staging (G) I-II and (H) III-IV and Rab1A expression. TNM, tumor-node-metastasis.

colon carcinoma, left colon carcinoma and rectal carcinoma tissues ( $\mathrm{P}>0.05$; Fig. 1C). Furthermore, Rab1A expression was increased in CRC tissues from tumor-node-metastasis (TNM) stage I-IV, although this change was not statistically significant $(\mathrm{P}>0.05$; Fig. 1E).

The relationship between RablA expression levels and clinicopathological parameters in CRC patients. We investigated the association between clinicopathological parameters and Rab1 A expression; the results were summarized in Table I. Rab1A expression was significantly associated with lymph node invasion $(\mathrm{P}<0.001$; Table $\mathrm{I})$, degree of differentiation $(\mathrm{P}<0.05$; Table I), venous invasion $(\mathrm{P}<0.05$; Table $\mathrm{I})$ and TNM stage $(\mathrm{P}<0.001$; Table I). However, no significant differences were observed between Rab1A expression and other clinicopathological variables such as age, sex, tumor size, neural invasion, tumor location or depth of tumor invasion (P>0.05; Table I).

RablA overexpression is associated with poor prognosis in $C R C$ patients. To explore the influence of high Rab1A expression on overall survival of CRC patients, we first analyzed the survival curves in CRC patients according to Rab1A expression level. The results revealed that the survival of patients with positive Rab1A expression was significantly worse than those with negative Rab1A expression ( $\mathrm{P}<0.001$; Fig. 2A). Subsequently, we performed subgroup analysis on lymph node invasion, tumor location and TNM staging. The results revealed that high Rab1A expression was associated with poor prognosis in lymph node invasion-negative patients $(\mathrm{P}<0.001$; Fig. 2B). A small but statistically insignificant difference was observed in lymph node invasion-positive patients ( $\mathrm{P}=0.054$; Fig. 2C). Furthermore, our results suggested that high expression of Rab1A was associated with poor prognosis in patients with right colon carcinoma and rectal cancer $(\mathrm{P}<0.001, \mathrm{P}<0.001$; Fig. 2D and $\mathrm{F})$, but not in patients with left colon carcinoma ( $\mathrm{P}=0.063$; Fig. $2 \mathrm{E})$. Moreover, higher Rab1 A expression was associated with a worse 5-year survival rate in patients with both TNM stages I-II and III-IV $(\mathrm{P}<0.001$, $\mathrm{P}=0.037$; Fig. 2G and $\mathrm{H}$ ).

Next, we performed univariate and multivariate analyses of RablA expression and clinicopathological variables. The univariate analysis revealed that Rab1A expression 
Table I. Association between Rab1A expression and clinicopathological factors in 142 patients with CRC.

\begin{tabular}{|c|c|c|c|}
\hline \multirow[b]{2}{*}{ Factors } & \multicolumn{3}{|c|}{ Rab1A } \\
\hline & Negative & Positive & $\mathrm{P}$-value \\
\hline \multicolumn{4}{|l|}{ Sex } \\
\hline Male & 31 & 58 & 0.727 \\
\hline Female & 20 & 33 & \\
\hline \multicolumn{4}{|l|}{ Age (years) } \\
\hline$\leq 60$ & 14 & 28 & 0.678 \\
\hline$>60$ & 37 & 63 & \\
\hline \multicolumn{4}{|l|}{ Size $(\mathrm{cm})$} \\
\hline$\leq 5$ & 33 & 46 & 0.103 \\
\hline$>5$ & 18 & 45 & \\
\hline \multicolumn{4}{|c|}{ Depth of invasion } \\
\hline $\mathrm{T} 1-2$ & 13 & 17 & 0.340 \\
\hline $\mathrm{T} 3-4$ & 38 & 74 & \\
\hline \multicolumn{4}{|c|}{ Lymph node invasion } \\
\hline Negative & 42 & 42 & $<0.001^{\mathrm{b}}$ \\
\hline Positive & 9 & 49 & \\
\hline \multicolumn{4}{|c|}{ Degree of differentiation } \\
\hline Well & 47 & 72 & $0.043^{\mathrm{a}}$ \\
\hline Poor & 4 & 19 & \\
\hline \multicolumn{4}{|c|}{ Venous invasion } \\
\hline Negative & 34 & 41 & $0.013^{\mathrm{a}}$ \\
\hline Positive & 17 & 50 & \\
\hline \multicolumn{4}{|c|}{ Neural invasion } \\
\hline Negative & 38 & 57 & 0.149 \\
\hline Positive & 13 & 34 & \\
\hline \multicolumn{4}{|c|}{ TNM staging } \\
\hline I-II & 41 & 42 & $<0.001^{\mathrm{b}}$ \\
\hline III-IV & 10 & 49 & \\
\hline \multicolumn{4}{|c|}{ Tumor location } \\
\hline Right & 19 & 29 & 0.777 \\
\hline Left & 15 & 31 & \\
\hline Rectum & 17 & 31 & \\
\hline
\end{tabular}

${ }^{\mathrm{a}} \mathrm{P}<0.05 ;{ }^{\mathrm{b}} \mathrm{P}<0.001$. CRC, colorectal cancer; TNM, tumor-node-metastasis.

$(\mathrm{P}<0.001$; Table II), lymph node invasion $(\mathrm{P}<0.001$; Table II), degree of differentiation ( $\mathrm{P}=0.005$; Table II), and TNM staging $(\mathrm{P}=0.001$; Table II) were related with poor prognosis. In the multivariate analysis, only Rab1A expression was confirmed as an independent prognostic factor for the survival of CRC patients $(\mathrm{P}<0.001$; Table II $)$.

Subgroup analysis of prognostic factors based on RablA expression level. In further investigations, we performed subgroup analysis of the relationship between Rab1A expression and survival time. The results revealed that Rab1A overexpression was associated with worse survival of CRC patients compared to those with low Rab1A expression, regardless of patient age, sex, tumor size, venous invasion, neural invasion or the TNM staging. Moreover, high Rab1A expression was associated with markedly shorter survival time in CRC patients with stage T3-T4 tumor depth $(\mathrm{P}<0.001$; Fig. 3), negative lymph node metastasis $(\mathrm{P}=0.001$; Fig. 3) and high-level differentiation ( $\mathrm{P}<0.001$; Fig. 3). In other words, patients with the depth of tumor invasion (stage T3-T4), lymph node metastasis (negative) or stage of differentiation (high-level), exhibited higher levels of RablA expression and were associated with poorer survival time. In contrast, there was no significant difference in survival time between patients with stage T1-T2 disease $(\mathrm{P}=0.265$; Fig. 3), positive lymph node metastasis $(\mathrm{P}=0.065$; Fig. 3$)$ or poor differentiation ( $\mathrm{P}=0.078$; Fig. 3) regardless of the Rab1A expression level.

High levels of p-S6K1 are closely related with RablA expression levels in CRC patients. Previous studies have shown that Rab1A is an activator of mTORC1 and HER2, which are the main upstream signaling molecules in the AKT/mTOR/S6K1 pathway. In the present study, we investigated the association between Rab1A expression and HER2/AKT/mTORC/S6K1 axis. Firstly, we performed IHC staining of p-S6K1, HER2 and p-AKT levels in 142 pairs of CRC and adjacent normal tissues. IHC analysis revealed high expression levels of p-S6K1 and p-AKT in CRC tissues compared with those in adjacent normal tissues $(\mathrm{P}<0.001, \mathrm{P}<0.05$; Fig. $4 \mathrm{D}$ and $\mathrm{E}$, and $4 \mathrm{G}$ and $\mathrm{H})$. There was no significant difference in HER2 expression levels between the CRC tissues and adjacent normal tissues $(\mathrm{P}>0.05$; Fig. 4A and B). Notably, the levels of HER2, p-AKT and p-S6K1 were positively associated with Rab1A expression in CRC tissues, particularly p-S6K1 $(\mathrm{P}<0.05, \mathrm{P}<0.01, \mathrm{P}<0.001$; Fig. 4C, 4F and I). In addition, Pearson $\chi^{2}$ test analysis revealed that Rab1A expression was closely related with the levels of HER2, p-AKT and p-S6K1 ( $\mathrm{P}=0.039, \mathrm{P}=0.001, \mathrm{P}<0.001$; Table III). In conclusion, high Rab1A expression levels were positively associated with the HER2/AKT/mTORC/S6K1 axis. Moreover, Rab1A expression was significantly associated with p-S6K1 levels.

Both high RablA and p-S6K1 levels are associated with a poor prognosis. We observed that high p-S6K1 levels were closely related with high Rab1A expression. We next generated a heat map displaying the expression levels of Rab1 A and p-S6K1 detected in 142 CRC patients through IHC analysis. Moreover, we compared the levels of Rab1A and p-S6K1 detected in 142 CRC tissues in a scatter plot of IHC scores. The results revealed that Rab1A expression was positively related to $\mathrm{p}-\mathrm{S} 6 \mathrm{~K} 1$ ( $\mathrm{P}<0.001$; Fig. $5 \mathrm{~A}$ and $\mathrm{B})$. The relationship between $\mathrm{p}-\mathrm{S} 6 \mathrm{~K} 1 / \mathrm{Rab} 1 \mathrm{~A}$ expression levels and clinicopathological variables was then explored; the results are summarized in Table IV. We concluded that high levels of both Rab1A and p-S6K1 were significantly related with tumor size ( $\mathrm{P}=0.046$; Table IV), lymph node invasion ( $\mathrm{P}<0.001$; Table IV), degree of differentiation ( $\mathrm{P}=0.012$; Table IV), venous invasion $(\mathrm{P}=0.002$; Table IV) and TNM stage $(\mathrm{P}<0.001$; Table IV) compared with low levels of either Rab1A or p-S6K1. No positive associations were obtained with other clinicopathological variables, such as age, sex, neural invasion, tumor location or depth of tumor invasion ( $\mathrm{P}>0.05$; Table IV). 
Table II. Results of univariate and multivariate analyses of the survival of patients with CRC by Cox's proportional hazard model.

\begin{tabular}{|c|c|c|c|c|c|c|}
\hline \multirow[b]{2}{*}{ Factors } & \multicolumn{3}{|c|}{ Univariate analysis } & \multicolumn{3}{|c|}{ Multivariate analysis } \\
\hline & HR & $95.0 \% \mathrm{CI}$ & P-value & HR & $95.0 \% \mathrm{CI}$ & P-value \\
\hline Sex (male/female) & 1.301 & $0.752-2.252$ & 0.347 & & & \\
\hline Age $(\leq 60$ or $>60$ years $)$ & 0.964 & $0.547-1.697$ & 0.899 & & & \\
\hline Size of cancer $(\leq 5$ or $>5 \mathrm{~cm})$ & 0.672 & $0.402-1.125$ & 0.131 & & & \\
\hline Depth of invasion (T1-2/T3-4) & 0.459 & $0.208-1.012$ & 0.054 & & & \\
\hline Lymph node metastasis (negative/positive) & 0.382 & $0.225-0.649$ & $<0.001^{\mathrm{b}}$ & 0.002 & $<0.001->10.000$ & 0.914 \\
\hline Degree of differentiation (poor/ well) & 0.432 & $0.239-0.781$ & $0.005^{\mathrm{a}}$ & 0.619 & $0.331-1.160$ & 0.134 \\
\hline Venous invasion (negative/ positive) & 0.772 & $0.461-1.294$ & 0.326 & & & \\
\hline Neural invasion (negative/positive) & 0.793 & $0.468-1.344$ & 0.389 & & & \\
\hline TNM stage (I-II/III-IV) & 0.394 & $0.231-0.670$ & $0.001^{\mathrm{a}}$ & $>10.000$ & $<0.001->10.000$ & 0.920 \\
\hline Rab1A expression (low/high) & 0.183 & $0.086-0.390$ & $<0.001^{\mathrm{b}}$ & 0.227 & 0.104-0.499 & $<0.001^{\mathrm{b}}$ \\
\hline
\end{tabular}

${ }^{\mathrm{a}} \mathrm{P}<0.01 ;{ }^{\mathrm{b}} \mathrm{P}<0.001 . \mathrm{CRC}$, colorectal cancer; TNM, tumor-node-metastasis.

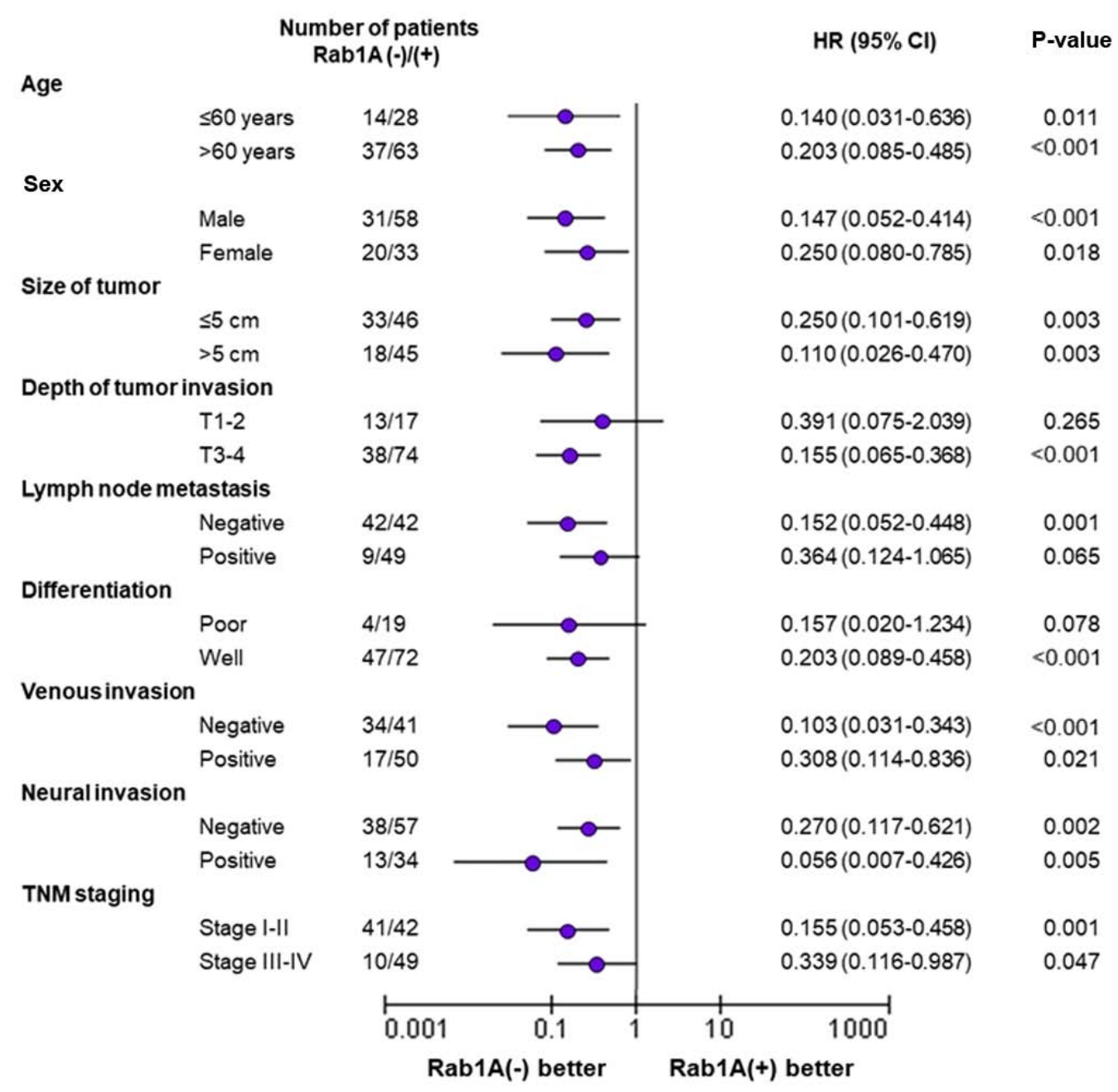

Figure 3. Subgroup analysis of the influence of Rab1 A expression on survival duration of CRC patients. Rab1A expression was negative in 31 male patients and positive in 58 male patients. Rab1A expression levels were associated with the survival duration in male patients $(\mathrm{P}<0.001)$ and in female patients $(\mathrm{P}=0.018)$. There were no significant differences in terms of variables such as depth of tumor invasion (T1-2), lymph node metastasis (positive) and differentiation (poor). CRC, colorectal carcinoma; TNM, tumor-node-metastasis.

We also explored the influence of the high levels of p-S6K1 on overall survival of patients with CRC by
Kaplan-Meier analysis. Compared with low p-S6K1 levels, high levels of $\mathrm{p}-\mathrm{S} 6 \mathrm{~K} 1$ were associated with a poorer 
A

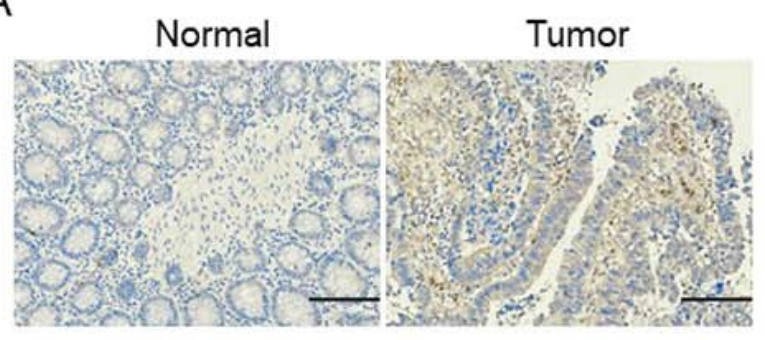

D

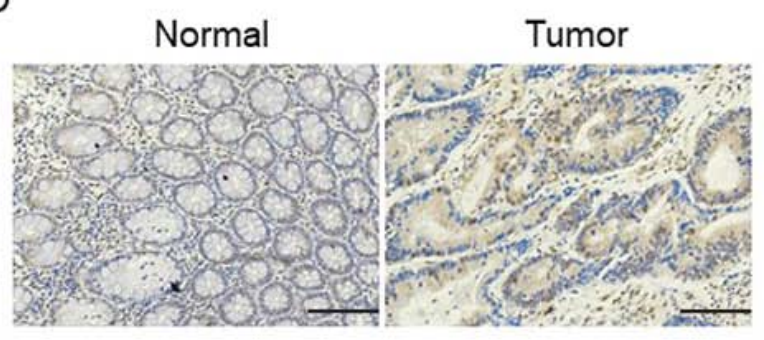

G

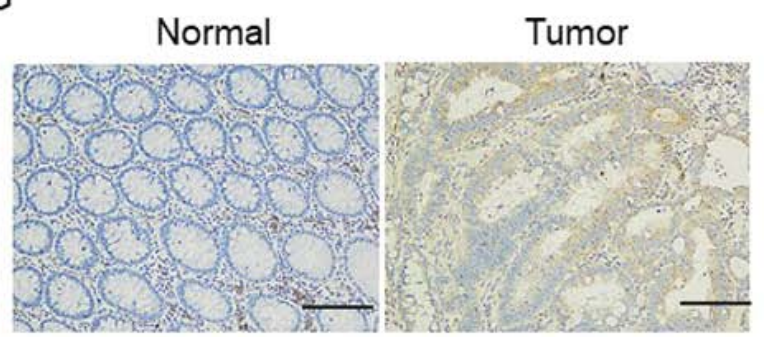

B

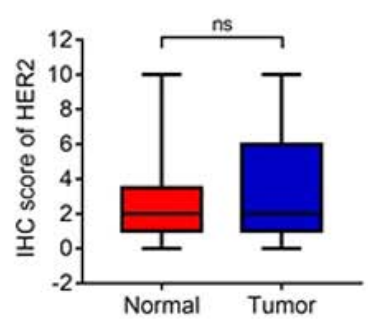

E

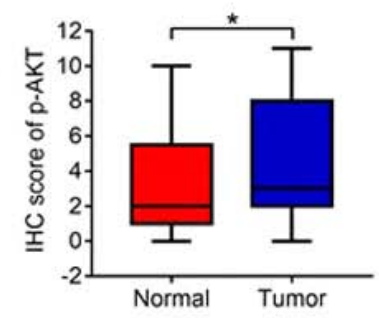

$\mathrm{H}$

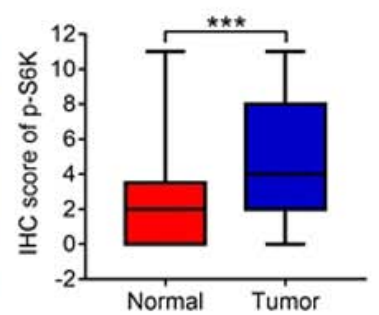

C

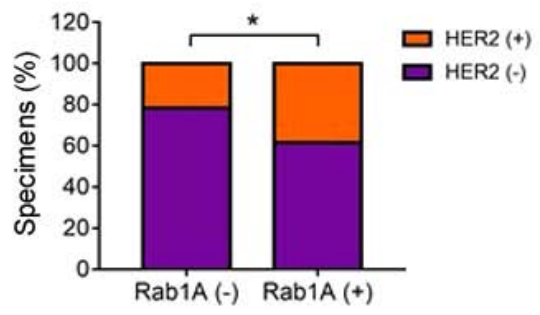

F

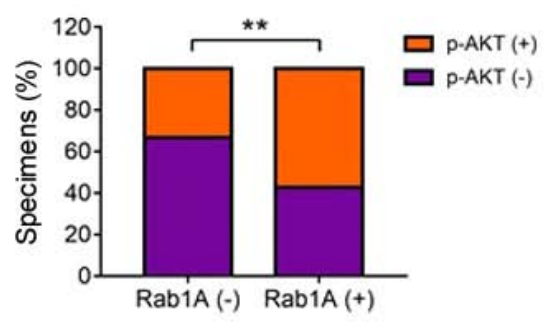

I

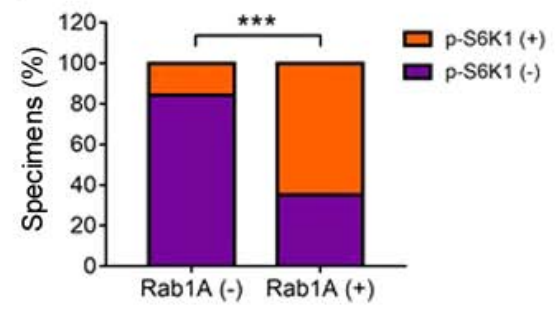

Figure 4. The relationship between Rab1A expression and HER2, p-AKT and p-S6K1 expression. Expression of (A) HER2, (D) p-AKT and (G) p-S6K1 was analyzed by immunohistochemical (IHC) staining of CRC and adjacent normal tissues (scale bar, $100 \mu \mathrm{m}$ ). IHC analysis of (B) HER2, (E) p-AKT and (H) p-S6K1 expression in tumor and normal tissues. Percentage of specimens exhibiting negative or positive Rab1A expression and association of Rab1A with (C) HER2, (F) p-AKT and (I) p-S6K1 in CRC tumor specimens. ${ }^{*} \mathrm{P}<0.05,{ }^{* *} \mathrm{P}<0.01,{ }^{* * *} \mathrm{P}<0.001$. CRC, colorectal carcinoma; ns, not significant.

Table III. Statistics of HER2/p-S6K1/p-AKT and Rab1A expression in CRC patients.

\begin{tabular}{lccc}
\hline Expression & $\begin{array}{c}\text { Rab1A } \\
\text { negative }\end{array}$ & $\begin{array}{c}\text { Rab1A } \\
\text { positive }\end{array}$ & P-value \\
\hline HER2 negative & 40 & 56 & $0.039^{\mathrm{a}}$ \\
HER2 positive & 11 & 35 & \\
p-S6K1 negative & 43 & 32 & $<0.001^{\mathrm{c}}$ \\
p-S6K1 positive & 8 & 59 & \\
p-AKT negative & 37 & 39 & $0.001^{\mathrm{b}}$ \\
p-AKT positive & 14 & 52 & \\
\hline
\end{tabular}

${ }^{\mathrm{a}} \mathrm{P}<0.05 ;{ }^{\mathrm{b}} \mathrm{P}<0.01 ;{ }^{\mathrm{c}} \mathrm{P}<0.001 . \mathrm{CRC}$, colorectal cancer.

prognosis in CRC patients $(\mathrm{P}<0.001$; Fig. 5C). Moreover, the survival of patients with high levels of both Rab1A and p-S6K1 was significantly worse than those with low levels of either Rab1A or p-S6K1 (P<0.001; Fig. 5D). Additionally, high levels of both Rab1A and p-S6K1 were associated with a poorer prognosis than high levels of either Rab1A or p-S6K1 alone $(\mathrm{P}=0.027$; Fig. 5E).
RablA expression in five colorectal cancer cell lines and sh-RNA-mediated deletion of the RablA gene in SW480 cells. We examined Rab1A expression levels in five of human colorectal cancer cell lines (HCT116, SW480, LOVO, COLO201 and RKO) by western blot analysis (Fig. 6A). Rab1A expression was relatively high in HCT116 and SW480 cells, and relatively low in LOVO cells. We next investigated the effects of shRNA-mediated-knockdown of Rab1A expression SW480 cells. Evaluation of the transfection efficiency by western blot analysis revealed that Rab1A expression was distinctly knocked down in cells transfected with RablA-shRNA compared to the expression in control-shRNA-transfected and untreated cells $(\mathrm{P}<0.05$; Fig. 6B and $\mathrm{C})$. These results confirmed effective and specific suppression of Rab1 A expression in SW480 cells.

RablA expression is positively related to $p$-S6K1 levels and regulates p-S6K1 in HCT116 cells and SW480 cells. To further clarify the relationship between Rab1A and p-S6K1 in HCT116 cells and SW480 cells, we examined the expression levels of p-S6K1, HER2 and p-AKT by western blotting after Rab1A knockdown. The results revealed that p-S6K1 levels were significantly decreased following Rab1A knockdown $(\mathrm{P}<0.05 ;$ Fig. $6 \mathrm{D}$ and $\mathrm{E})$, while there was no significant change in the levels of HER2 and p-AKT (P>0.05; Fig. 6D and E), 
A
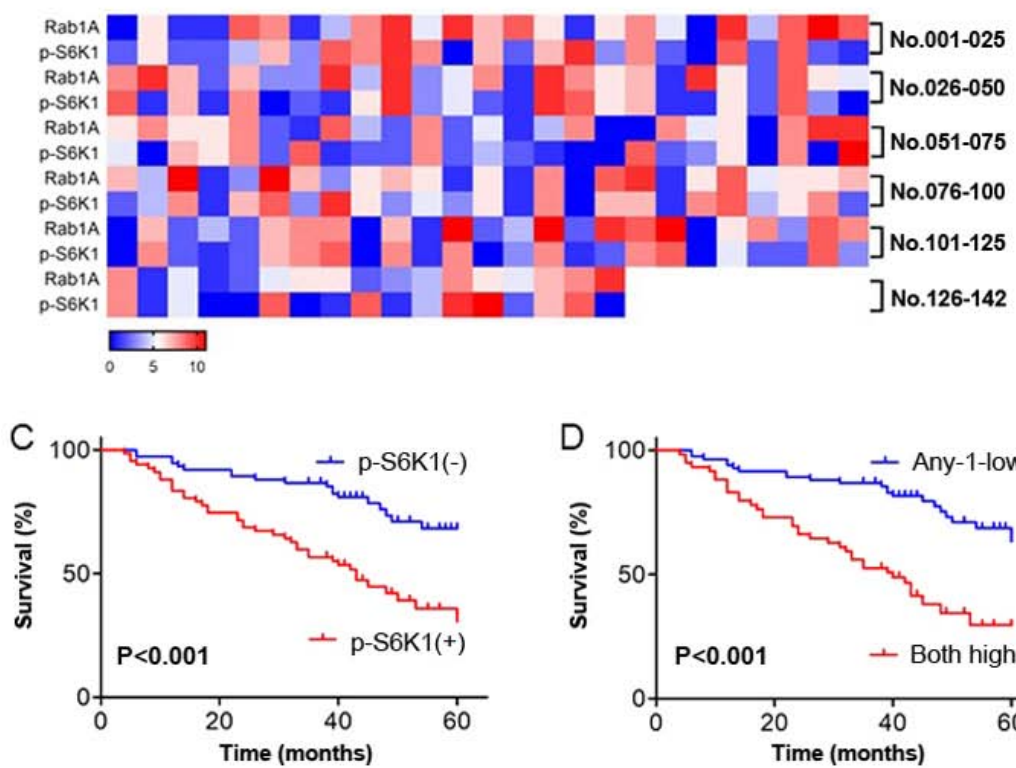

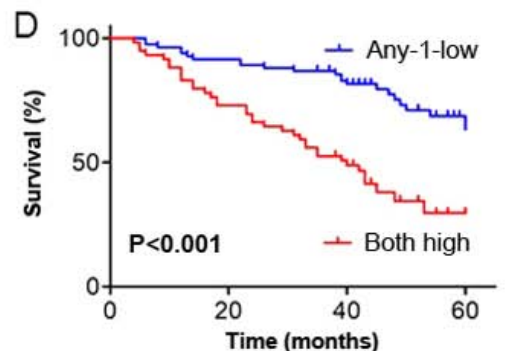

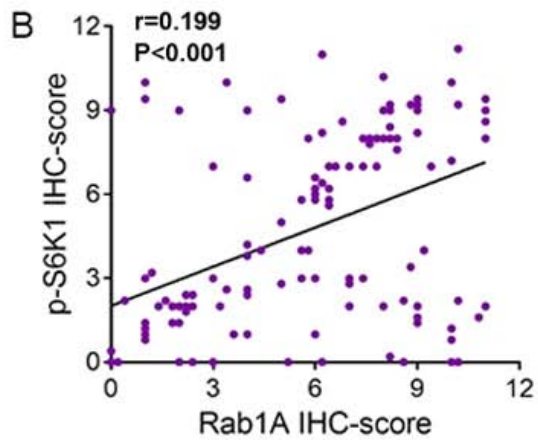

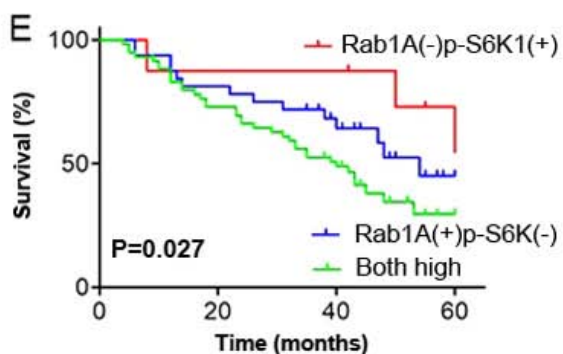

Figure 5. Analysis of the associations between the expression of Rab1A and p-S6K1. (A) The association between Rab1A and p-S6K1 expression in 142 CRC tissues displayed as a heat map. (B) Scatter plot analysis of the relationship between Rab1A and p-S6K1 expression based on average IHC scores of five randomly selected fields-of-view and a non-parametric Spearman test was used. (C) Kaplan-Meier survival analysis of 142 CRC patients after surgery according to p-S6K1 expression. (D) Survival analysis of patients with high expression of Rab1A and p-S6K1 and low expression of either Rab1A or p-S6K1. (E) Survival analysis of patients with co-overexpression of Rab1A and p-S6K1 and patients with overexpression of Rab1A or p-S6K1 alone. CRC, colorectal carcinoma; IHC, immunohistochemical; CTL, untreated; NC, negative control shRNA; KD, shRab1A.

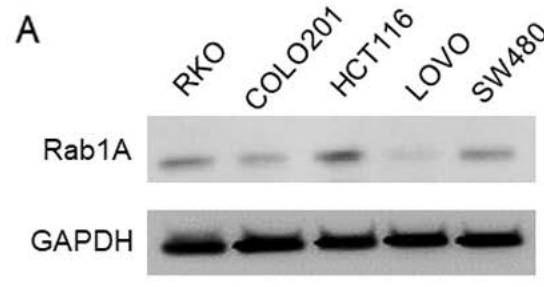

B
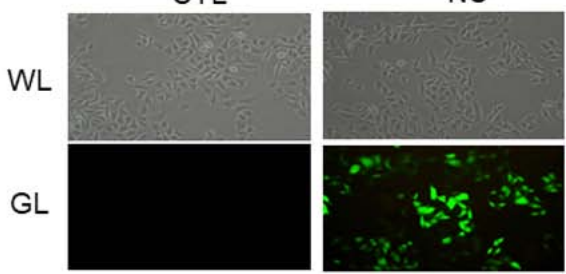

D

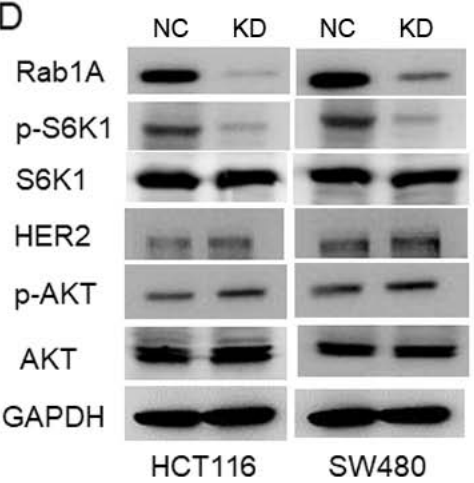

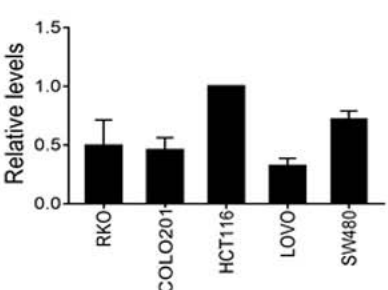

KD

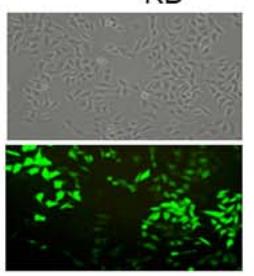

$\mathrm{E}$

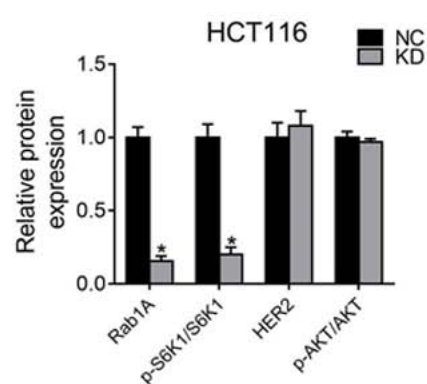

C
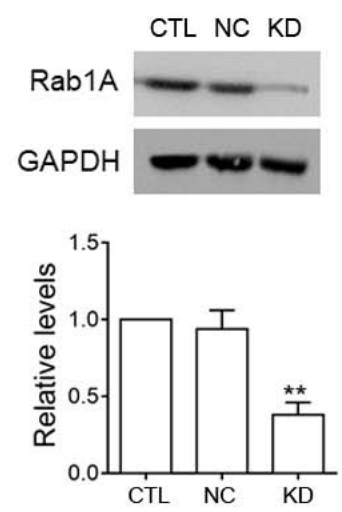

SW480

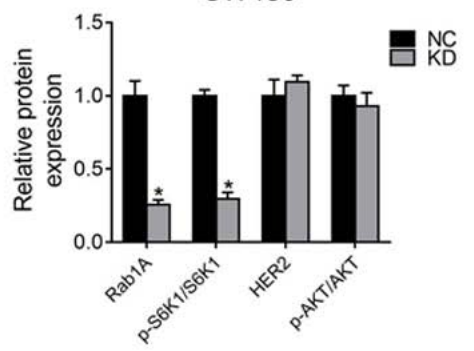

Figure 6. Rab1 A expression in five colorectal cancer cell lines (HCT116, SW480, LOVO, COLO201 and RKO) and the relationship between Rab1A and p-S6K1 expression in HCT116 cells and SW480 cells. (A) Western blot analysis of Rab1A expression levels in five colorectal cancer cell lines. The intensity of the bands was determined by densitometric analysis. (B) Fluorescence microscopic analysis of the transfection efficiency in the untreated (CTL), shRab1A (KD) and negative control shRNA (NC) groups. (C) Western blot analysis of Rab1 A expression in the shRab1A (KD), negative control shRNA (NC) and untreated (CTL) groups. The results were quantitatively analyzed based on grayscale values. (D) The protein levels of Rab1A/GAPDH, HER2/GAPDH, p-AKT/AKT and p-S6K1/S6K1 were detected by western blotting in the SW480 cells/HCT116 cells transfected with shRab1A (KD) and negative control shRNA (NC). (E) The results were quantitatively analyzed with gray values in HCT116 cells and SW480 cells. ${ }^{*} \mathrm{P}<0.05,{ }^{{ }^{* *} \mathrm{P}}<0.01$. 

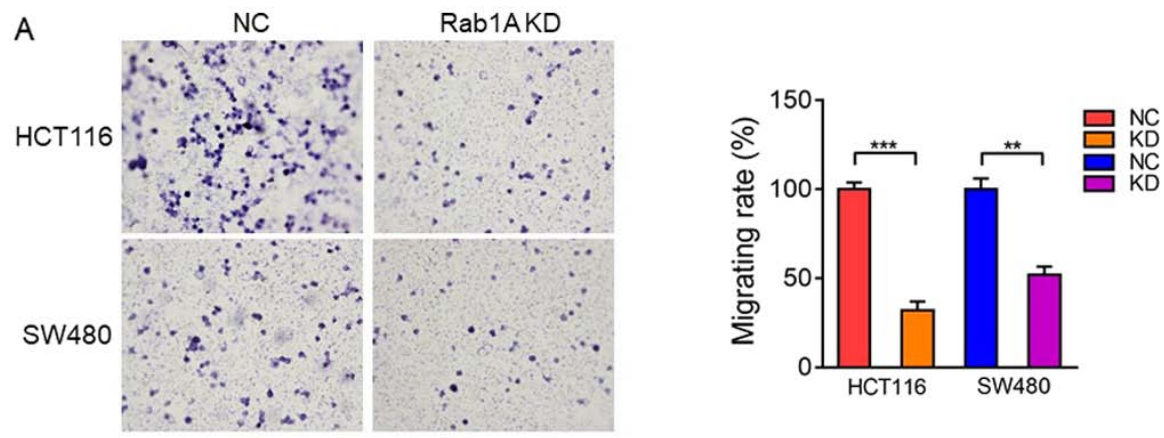

B

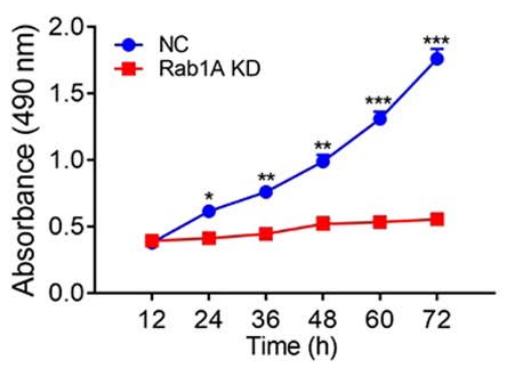

C

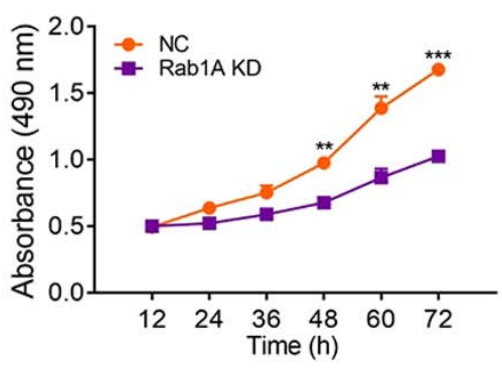

Figure 7. Effects of Rab1A expression on migration and proliferation of colorectal cancer cell lines. (A) Transwell migration assay analysis of the migration ability of SW480 and HCT116 cells transfected with negative control shRNA (NC) or shRab1A (KD). The migration rate was analyzed based on the relative number of migratory cells; data represent the mean \pm standard error of the mean (SEM) ( $=5$ ). Representative images are presented (magnification, $\mathrm{x} 200)$. (B and C) MTT assay analysis of the proliferation ability of SW480 and HCT116 cells transfected with negative control shRNA (NC) or shRab1A (KD); data represent the mean $\pm \operatorname{SEM}(\mathrm{n}=3) .{ }^{*} \mathrm{P}<0.05,{ }^{* *} \mathrm{P}<0.01,{ }^{* * * *} \mathrm{P}<0.001$.

indicating that Rab1 A expression is positively related with p-S6K1 levels but has no effect on the HER2/AKT/mTORC1 axis. To sum up, we concluded that Rab1A expression is not only closely related to $\mathrm{p}-\mathrm{S} 6 \mathrm{~K} 1$ levels, but also mediates targeted regulation of p-S6K1 in HCT116 and SW480 cells.

RablA knockdown inhibits the migration and proliferation of HCT116 and SW480 cells. We explored the effect of Rab1A on migration and proliferation of HCT116 and SW480 cells in vitro. Transwell migration assays revealed that the migration ability of SW480 and HCT116 cells was significantly inhibited following Rab1A knockdown ( $\mathrm{P}<0.05$; Fig. 7A). Furthermore, MTT assays performed at 12, 24, 36, 48, 60 and $72 \mathrm{~h}$ after transfection revealed that the proliferation of HCT116 and SW480 cells transfected with Rab1A-shRNA was significantly inhibited compared with that of the cells transfected with control-shRNA $(\mathrm{P}<0.05$; Fig. 7B and $\mathrm{C})$.

\section{Discussion}

Colorectal cancer is an important health problem worldwide (22). Despite the development of various diagnostic and treatment strategies, including chemotherapy, the prognosis is poor and the survival rate is low (23). The HER2/AKT/mTOR/S6K1 pathway plays a significant role in $\mathrm{CRC}$. The emergence of rapamycin has prolonged the survival rate in CRC modestly, but diverse side-effects and severe drug-resistance remain challenges. Therefore, identification of novel therapeutic targets for CRC are urgently required $(24,25)$, particularly for mTORC1-targeted therapy-resistant cancers. Based on this perspective, we investigated the expression patterns and association between Rab1A and p-S6K1 and their clinical significance in CRC patients. Moreover, we validated the anticancer effects of Rab1A expression by shRNA-mediated knockdown in CRC cells.

Rab1A is a small GTPase well known for its role in regulating ER-to-Golgi vesicular transport (26). Rab1A has recently been reported to function as an oncogene and is overexpressed in various types of cancers, such as tongue squamous carcinoma (27), cervical (28), breast (10) and prostate cancer $(29,30)$, hepatocellular carcinomas $(11)$, and lung cancer (31). In our present study, we first investigated the Rab1A expression status and evaluated its clinical significance and relationship with overall survival. Our results revealed that Rab1A expression levels were significantly higher in CRC tissues than in paracancerous tissues, and higher in patients with lymph node invasion than in those without. Furthermore, Rab1A expression increased as TNM staging progressed from I to IV in CRC, although the relationship was not statistically significant. However, there were no significant differences in Rab1A expression between right colon carcinoma, left colon carcinoma and rectal carcinoma, indicating that Rab1A expression is not related to tumor location. High Rab1A expression was significantly associated with several clinicopathological parameters such as lymph node invasion, poor degree of differentiation, venous invasion and high TNM stage.

Previous studies have demonstrated that Rab1A overexpression leads to poor survival in HCC (11) and gastric cancer (32). In accordance with a previous study in HCC (11), we found that the survival of Rab1A-positive patients was significantly worse than that of Rab1A-negative patients and higher Rab1A expression was associated with a worse 5-year survival rate in patients with I-II and III-IV disease staging. 
Table IV. Association between Rab1A/p-S6K1 positive or negative and clinicopathological factors in 142 patients with CRC.

\begin{tabular}{|c|c|c|c|}
\hline \multirow[b]{2}{*}{ Factors } & \multicolumn{2}{|c|}{ Rab1A and p-S6K1 } & \multirow[b]{2}{*}{ P-value } \\
\hline & Any-1-low & Both high & \\
\hline \multicolumn{4}{|l|}{ Sex } \\
\hline Male & 54 & 35 & 0.486 \\
\hline Female & 29 & 24 & \\
\hline \multicolumn{4}{|c|}{ Age (years) } \\
\hline$\leq 60$ & 25 & 17 & 0.866 \\
\hline$>60$ & 58 & 42 & \\
\hline \multicolumn{4}{|l|}{ Size $(\mathrm{cm})$} \\
\hline$\leq 5$ & 52 & 27 & $0.046^{\mathrm{a}}$ \\
\hline$>5$ & 31 & 32 & \\
\hline \multicolumn{4}{|c|}{ Depth of invasion } \\
\hline $\mathrm{T} 1-2$ & 19 & 11 & 0.541 \\
\hline T3-4 & 64 & 48 & \\
\hline \multicolumn{4}{|c|}{ Lymph node invasion } \\
\hline Negative & 65 & 19 & $<0.001^{\mathrm{c}}$ \\
\hline Positive & 18 & 40 & \\
\hline \multicolumn{4}{|c|}{ Degree of differentiation } \\
\hline Well & 75 & 44 & $0.012^{\mathrm{a}}$ \\
\hline Poor & 8 & 15 & \\
\hline \multicolumn{4}{|c|}{ Venous invasion } \\
\hline Negative & 53 & 22 & $0.002^{\mathrm{b}}$ \\
\hline Positive & 30 & 37 & \\
\hline \multicolumn{4}{|c|}{ Neural invasion } \\
\hline Negative & 59 & 36 & 0.209 \\
\hline Positive & 24 & 23 & \\
\hline \multicolumn{4}{|c|}{ TNM staging } \\
\hline I-II & 64 & 19 & $<0.001^{\mathrm{c}}$ \\
\hline III-IV & 19 & 40 & \\
\hline \multicolumn{4}{|c|}{ Tumor location } \\
\hline Right & 33 & 15 & 0.170 \\
\hline Left & 23 & 23 & \\
\hline Rectum & 27 & 21 & \\
\hline
\end{tabular}

${ }^{\mathrm{a}} \mathrm{P}<0.05 ;{ }^{\mathrm{b}} \mathrm{P}<0.01 ;{ }^{\mathrm{c}} \mathrm{P}<0.001 . \mathrm{CRC}$, colorectal cancer; TNM, tumor-lymph node metastasis.

Furthermore, univariate and multivariate analysis indicated that Rab1A expression was the only independent prognostic factor for CRC patients, which is consistent with the results reported by Wang et al (33). Subgroup analysis of the relationships of high/low Rab1A expression levels with other prognostic factors revealed that Rab1A overexpression was associated with poorer survival of CRC patients compared with that associated with low Rab1A expression, regardless of patient age, sex, tumor size, venous invasion, neural invasion and TNM staging. These findings suggest that high Rab1A levels play a significant role in the prognosis of CRC.
Previous studies have demonstrated that Rab1A functions as an activator of mTORC1 in CRC, prostate cancer and HCC $(11,12,29)$. In addition to the function of Rab1 A upstream of mTORC1, it is possible that Rab1A, which is involved in autophagy, influences downstream molecules, such as the other autophagic small GTPases Rab7 (34) and Rab12 (35), which are regulated by mTORC1/ULK. HER 2 is the main upstream signaling molecule in the AKT/mTOR/S6K1 pathway and p-S6K1 is downstream of mTORC1 (36). Based on this information, we first reported the associations between Rab1A and upstream or downstream significant targets of mTORC1 such as p-S6K1, Her-2 and p-AKT in CRC patients. IHC analysis revealed that high Rab1A expression was positively related with the HER2/AKT/mTORC/S6K1 axis, although no significant relationship between Rab1A expression and the HER2/AKT/mTORC axis was identified by western blot analysis. Notably, Rab1A expression was found to be closely associated with p-S6K1 levels both by IHC and western blot analysis, suggesting that Rab1 A is positively associated with p-S6K1 rather than the HER2/AKT/mTORC axis. Furthermore, high levels of both p-S6K1 and Rab1A were related with several clinicopathological factors such as size, lymph node invasion, and degree of differentiation. Moreover, we first reported that high levels of both Rab1A and p-S6K1 were associated with a worse prognosis compared with high levels of Rab1 A or p-S6K1 alone. These findings indicated that high levels of both p-S6K1 and Rab1A had significantly more detrimental effects on the prognosis of patients with CRC compared with the effect of high levels of Rab1A or p-S6K1 alone. Our in vitro studies indicated that p-S6K1 levels sharply decreased after Rab1A knockdown rather than p-AKT/HER-2. We searched the relevant data of RAB1A, HER2, AKT, mTOR, S6K1 and analyzed the association in TCGA dataset via GEPIA platform. It revealed that Rab1A was an activator of mTORC1 and closely related with mTOR and p-S6K1. However, RAB1A was also moderately correlated with AKT, and not related with HER-2. It suggested that Rab1A activated the mTORC1 pathway independent of HER2 (data not shown). In conclusion, Rab1 A was not only closely related with p-S6K1 levels, but also targeted regulation of p-S6K1, rather than the HER2/AKT/mTORC axis.

Rab1A overexpression in HCC cell lines and Rab1A silencing has been revealed to significantly inhibit the growth and migration abilities of HCC cell lines (11). In our comparisons of five different HCC cell lines, Rab1A expression was relatively high in HCT116 and SW480 cells, and relatively low in LOVO cell lines. In accordance with the results in HCC tissues, knockdown of Rab1A expression inhibited the migration and proliferation of HCT116 cells and SW480 cells, suggesting that Rab1A promotes viability and migration abilities via regulation of the HER2/AKT-independent mTOR/S6K1 pathway in colorectal cancer. However, some limitations exist in our study, such as the specific mechanism of how Rab1A activates mTOR independently of HER2/AKT. Thus, further research is required to clarify the specific regulatory mechanisms between Rab1A and HER2/AKT.

In conclusion, our results revealed that Rab1A expression was increased in CRC tissues. Overexpression of Rab1A was related with clinicopathological parameters and indicated a poor prognosis. Furthermore, high levels of p-S6K1 were 
detected in CRC tissues and RablA expression had a positive association with p-S6K1 levels. In addition, high levels of both Rab1A and p-S6K1 were associated with a poorer prognosis compared with high levels of either Rab1 A or p-S6K1 alone. Moreover, we determined that Rab1A knockdown significantly reduced the migration and proliferation abilities of CRC cell lines by targeting regulation of $\mathrm{p}-\mathrm{S} 6 \mathrm{~K} 1$. Thus, our study revealed that Rab1A plays an important role in CRC and may provide a new perspective on targeted therapy of CRC, particularly for mTORC1-targeted therapy-resistant cancers.

\section{Acknowledgements}

Not applicable.

\section{Funding}

This study was supported by the Beijing Medical and Health Foundation (B17523).

\section{Availability of data and materials}

The datasets used during the present study are available from the corresponding author upon reasonable request.

\section{Authors' contributions}

JM, JuW and CZ conceived and designed the experiments; ZC, JiW, MX, XK, LZ and XS performed the experiments; ZC, $\mathrm{XS}, \mathrm{XK}$ and LZ collected and analyzed the data; ZC, JuW and JiW revised the manuscript. All authors read and approved the manuscript and agree to be accountable for all aspects of the research in ensuring that the accuracy or integrity of any part of the work are appropriately investigated and resolved.

\section{Ethics approval and consent to participate}

This study was approved by the Independent Ethics Committee of the Affiliated Suzhou Hospital of Nanjing Medical University (Suzhou, China) and all participants provided written informed consent.

\section{Patient consent for publication}

Not applicable.

\section{Competing interests}

The authors state that they have no competing interests.

\section{References}

1. Siegel RL, Miller KD and Jemal A: Cancer statistics, 2018. CA Cancer J Clin 68: 7-30, 2018.

2. Ng SC and Wong SH: Colorectal cancer screening in Asia. $\mathrm{Br}$ Med Bull 105: 29-42, 2013.

3. DeSantis CE, Lin CC, Mariotto AB, Siegel RL, Stein KD, Kramer JL, Alteri R, Robbins AS and Jemal A: Cancer treatment and survivorship statistics, 2014. CA Cancer J Clin 64: 252-271, 2014.

4. Hutagalung AH and Novick PJ: Role of Rab GTPases in membrane traffic and cell physiology. Physiol Rev 91: 119-149, 2011.
5. Charng WL, Yamamoto S, Jaiswal M, Bayat V, Xiong B, Zhang K, Sandoval H, David G, Gibbs S, Lu HC, et al: Drosophila Tempura, a novel protein prenyltransferase $\alpha$ subunit, regulates notch signaling via Rab1 and Rab11. PLoS Biol 12: e1001777, 2014.

6. Wang C, Yoo Y,Fan H, Kim E, Guan KL and Guan JL: Regulation of Integrin $\beta 1$ recycling to lipid rafts by Rabla to promote cell migration. J Biol Chem 285: 29398-29405, 2010.

7. Tanaka M, Mun S, Harada A, Ohkawa Y, Inagaki A, Sano S, Takahashi K, Izumi Y, Osada-Oka M, Wanibuchi H, et al: Hsc70 contributes to cancer cell survival by preventing Rab1 A degradation under stress conditions. PLoS One 9: e96785, 2014.

8. Coune PG, Bensadoun JC, Aebischer P and Schneider BL: Rab1A over-expression prevents Golgi apparatus fragmentation and partially corrects motor deficits in an alpha-synuclein based rat model of Parkinson's disease. J Parkinsons Dis 1: 373-387, 2011.

9. Wu G, Yussman MG, Barrett TJ, Hahn HS, Osinska H, Hilliard GM, Wang X, Toyokawa T, Yatani A, Lynch RA, et al: Increased myocardial Rab GTPase expression: A consequence and cause of cardiomyopathy. Circ Res 89: 1130-1137, 2001.

10. Xu H, Qian M, Zhao B, Wu C, Maskey N, Song H, Li D, Song J, Hua K and Fang L: Inhibition of RAB1A suppresses epithelial-mesenchymal transition and proliferation of triple-negative breast cancer cells. Oncol Rep 37: 1619-1626, 2017.

11. Xu BH, Li XX, Yang Y, Zhang MY, Rao HL, Wang HY and Zheng XF: Aberrant amino acid signaling promotes growth and metastasis of hepatocellular carcinomas through RablA-dependent activation of mTORC1 by Rab1A. Oncotarget 6: 20813-20828, 2015.

12. Thomas JD, Zhang YJ, Wei YH, Cho JH, Morris LE, Wang HY and Zheng XF: Rab1A is an mTORC1 activator and a colorectal oncogene. Cancer Cell 26: 754-769, 2014.

13. Wullschleger $\mathrm{S}$, Loewith $\mathrm{R}$ and Hall $\mathrm{MN}$ : TOR signaling in growth and metabolism. Cell 124: 471-484, 2006.

14. Wang X, Chu Y, Wang W and Yuan W: mTORC signaling in hematopoiesis. Int J Hematol 103: 510-518, 2016.

15. Laplante M and Sabatini DM: mTOR signaling in growth control and disease. Cell 149: 274-293, 2012.

16. Shaw RJ and Cantley LC: Ras, PI(3)K and mTOR signalling controls tumour cell growth. Nature 441: 424-430, 2006.

17. Han G, Gong H, Wang Y,GuoS andLiu K: AMPK/mTOR-mediated inhibition of survivin partly contributes to metformin-induced apoptosis in human gastric cancer cell. Cancer Biol Ther 16: 77-87, 2015.

18. Don AS and Zheng XF: Recent clinical trials of mTOR-targeted cancer therapies. Rev Recent Clin Trials 6: 24-35, 2011.

19. Li L, Kim E, Yuan H, Inoki K, Goraksha-Hicks P, Schiesher RL, Neufeld TP and Guan KL: Regulation of mTORC1 by the Rab and Arf GTPases. J Biol Chem 285: 19705-19709, 2010.

20. Yang K, Jiang L, Hu Y, Yu J, Chen H, Yao Y and Zhu X: Short hairpin RNA- mediated gene knockdown of FOXM1 inhibits the proliferation and metastasis of human colon cancer cells through reversal of epithelial-to-mesenchymal transformation. J Exp Clin Cancer Res 34: 40, 2015.

21. Shao X, Kuai X, Pang Z, Zhang L, Wu L, Xu L and Zhou C: Correlation of Gli1 and HER2 expression in gastric cancer: Identification of novel target. Sci Rep 8: 397, 2018.

22. Zhou WW, Chu YP and An GY: Significant difference of neutrophil-lymphocyte ratio between colorectal cancer, adenomatous polyp and healthy people. Eur Rev Med Pharmacol Sci 21: 5386-5391, 2017.

23. Zhang Z, Dong X, Yang X, Wan D, Sun L, Gu M, Li M, Zhu Z, Wang J, Shang Z, et al: Expression and clinical significance of absent in melanoma 2 in colorectal cancer. Biomed Pharmacother 94: 843-849, 2017.

24. Polivka J Jr and Janku F: Molecular targets for cancer therapy in the PI3K/AKT/mTOR pathway. Pharmacol Ther 142: 164-175, 2014.

25. Phyu SM, Tseng CC, Fleming IN and Smith TA: Probing the PI3K/Akt/mTor pathway using 31P-NMR spectroscopy: Routes to glycogen synthase kinase 3. Sci Rep 6: 36544, 2016.

26. Satoh A, Wang Y, Malsam J, Beard MB and Warren G: Golgin-84 is a rab1 binding partner involved in Golgi structure. Traffic 4: $153-161,2003$.

27. Shimada K, Uzawa K, Kato M, Endo Y, Shiiba M, Bukawa H, Yokoe H, Seki N and Tanzawa H: Aberrant expression of RAB1A in human tongue cancer. Br J Cancer 92: 1915-1921, 2005.

28. Nikoshkov A, Broliden K, Attarha S, Sviatoha V, Hellström AC, Mints $M$ and Andersson S: Expression pattern of the PRDX2, RAB1A, RAB1B, RAB5A and RAB25 genes in normal and cancer cervical tissues. Int J Oncol 46: 107-112, 2015. 
29. Sun T, Wang X, He HH, Sweeney CJ, Liu SX, Brown M, Balk S, Lee GS and Kantoff PW: MiR-221 promotes the development of androgen independence in prostate cancer cells via downregulation of HECTD2 and RAB1A. Oncogene 33: 2790-2800, 2014.

30. Abd Elmageed ZY, Yang Y, Thomas R, Ranjan M, Mondal D, Moroz K, Fang Z, Rezk BM, Moparty K, Sikka SC, et al: Neoplastic reprogramming of patient-derived adipose stem cells by prostate cancer cell-associated exosomes. Stem Cells 32: 983-997, 2014

31. Wang X, Liu F, Qin X, Huang T, Huang B, Zhang Y and Jiang B Expression of Rab1A is upregulated in human lung cancer and associated with tumor size and T stage. Aging (Albany NY) 8: 2790-2798, 2016.

32. Xu B, Huang C, Yang X, Li X, Li L and Ding Y: Significance and prognostic role of human epidermal growth factor receptor 2 and RAB1A expression in gastric cancer. Oncol Lett 15: 5185-5192, 2018.

33. Wang ZK, Cheng ZW, Chen SJ, Zhu XG, Gu YP, Yang XD, Sun L, Liu WT, Zhang YJ, Yuan JF, et al: Aberrant expression of Rab1A and its prognostic significance in human colorectal cancer. Eur Rev Med Pharmacol Sci 22: 4509-4517, 2018.
34. Kim YM, Jung CH, Seo M, Kim EK, Park JM, Bae SS and Kim DH: mTORC1 phosphorylates UVRAG to negatively regulate autophagosome and endosome maturation. Mol Cell 57: 207-218, 2015.

35. Xu J, Fotouhi M and McPherson PS: Phosphorylation of the exchange factor DENND3 by ULK in response to starvation activates Rab12 and induces autophagy. EMBO Rep 16: 709-718, 2015.

36. Vaira V, Lee CW, Goel HL, Bosari S, Languino LR and Altieri DC: Regulation of survivin expression by IGF-1/mTOR signaling. Oncogene 26: 2678-2684, 2007.

(i) (9) This work is licensed under a Creative Commons Attribution-NonCommercial-NoDerivatives 4.0 International (CC BY-NC-ND 4.0) License. 